\title{
RULE-BASED INFERENCING SYSTEM FOR INFERTILITY DIAGNOSIS IN WOMEN
}

\author{
Khumukcham Robindro and Kshetrimayum Nilakanta \\ Department of Computer Science, Manipur University, Canchipur, India
}

\begin{abstract}
Childlessness among married couples is a rising problem in India. One of the major factors of childlessness is due to being infertile of either one or both of wife or husband. Infertility refers to the failure of a couple to become pregnant after one year of regular unprotected sexual intercourse. Infertility is a life crisis with invisible losses, and its consequences are manifold. This paper is intended to propose a rule-based inferencing of infertility diagnosis of women using Java Expert System Shell (JESS). Such a system is essential because the percentage of childlessness due to infertility is rising very high these days in India. This framework is aimed to enhance the existing tools used to identify and diagnose infertility problems in Women in the state of Manipur. We have implemented here the user interface component using Java, the knowledge base of the system using the Java Expert System Shell (JESS) and the Java IDE of Netbeans 7.0 while the database component is using SQL. The proposed framework can be used as guidelines for infertility diagnosis for women to assists the physicians with their daily practices and women who had infertility problems.
\end{abstract}

\section{KEYWORDS}

Childlessness; Infertility; Inferencing; Knowledge-Base; JESS

\section{INTRODUCTION}

Childlessness among married couples in India is on the rise. It has been raised by 50 percent from 1981 to 2001 among Indian couples [1] and is also a rising issue among the newly married couples due to being infertile of either one or both of wife or husband. Infertility has become common major issue these days and about 15 per cent couples are suffering from infertility in India [2]. In the last five years, infertility rates in India have been raised to 20-30 percent as per the researcher's data [3,4]. It has been known that studies related to infertility have been neglected in the past few decades instead more emphasis was placed on controlling the unwanted fertility. Infertility rate increases with increasing levels of educational attainment among women [5]. This can be related to the fact that with aspirations for attaining the higher educational level, marriage is delayed as a result of which in confirmation with aforementioned causation factors like higher age at marriage, urban living style etc. The prevalence of infertility is being widespread and reach to such a proportion that it has become as one of the leading public health problem affecting the life of whole society. Infertility, compounded by pregnancy wastage, infant and child mortality, may lead to depopulation. This poses a serious threat to the social and economic development [6]. It imposes a profound and social stress especially among women which in turn evoke the feelings of denial, anger, grief and guilt. 
Infertility affects a relatively large number of couples in their reproductive lives at some point in their reproductive lives both globally as well as in India [7]. It has become a source of concern in India very lately. According to a 2013 World Bank estimate, the drop in fertility started about 10 years ago in India, with a steady 17 percent decline from the year 2000, doctors told The Times of India and it is believed that 10 percent of the general population suffers from some form of infertility [8]. It is also found that there are many factors of causing infertility in women and the ethologist of infertility varies from region to region and from one population to another. The study on [9] has shown us that the prevalence of infertility is highest in the southern region of India and lowest in the northern region. According to the IIPS study, Tamil Nadu fared the worst among Indian states with almost 11 per cent of married women being childless and Haryana the best with only 2.52 percent childlessness compared to the national average of 6.3 percent. Chennai, Hyderabad and Chandel district in Manipur could be considered the 'infertility hubs' with one in five women being childless. In [10], the researcher shows in their study shows that West Bengal and Bihar top the list of childless women with 14.1 and 12.4 percent respectively as compared to the national average of 8.2 percent while Meghalaya has the lowest figure of 2.3 percent.

Infertility problems among women in Manipur have become a widespread issue now, which cause mental stress and financial burdens in the family. The study on $[11,12,13]$ discussed that there are various factors influencing fertility in Manipur. Age at marriage and age at first conception are two direct factors that influence women fertility. It also reveals that biological and social factors like age at menarche, age at marriage, age at first conception, women's education have an inverse relationship with a number of conceptions and live births whereas education of husband and type of family are inversely proportional with live birth only $[14,15,16]$. There is a relationship between the fertility pattern with education and occupation is studied in [17]. In this study, with regards to fertility performance, the highest number of ever born live birth is observed among the Nepalese and lowest among the Meiteis. There are many women from Manipur moves for infertility treatment outside the state like the cities of Guwahati, Kolkata, Mumbai and Delhi etc. According to the record, it is found that $60 \%$ of infertility women from the north-eastern region of India in a particular hospital of Guwahati excluding Assam are from Manipur, which is an alarming call to the Manipur society [18]. There could be many thousands of Manipuri women suffering from infertility problems other than those gone for infertility treatment outside the state. Besides these, many women are also moving to Delhi, Kolkata, Mumbai, etc. to seek medical help for infertility treatments whereas some are undergoing treatment in Manipur itself.

Infertility is defined as the inability of a couple to conceive following 12-24 months of regular unprotected sexual intercourse. The problem of infertility has not given its due attention in India because it is not a life-threatening condition. Infertility is a life crisis with invisible losses and its consequences are manifold [19]. It remains an ongoing global challenge, particularly for women living in low-resource settings. It is important for the global reproductive health community to engage in three forms of 21st-century activism: (i) address the preventable causes of infertility; (ii) provide supports and alternatives for the infertile, especially in resource-poor settings where parenthood is socially mandatory and (iii) make common cause with the growing LCIVF movement, which seeks reproductive justice for those living with infertility around the globe [20]. Married couples generally wanted to start a family happily right after the marriage and don't even imagine that they will be facing any difficulties while conceiving. Worldwide prevalence of infertility varies between 8-12\% among reproductive-aged couples [21]. In countries like India and other developing countries where the problem of population explosion remains at the top, 
issues of infertility also simultaneously grabbing the countries' population. In [22], it is discussed that there are emotional and psychological consequences of infertility among infertile women in the society and they are seeking treatment.

With an alarming size of the population affected by infertility issues and to avoid the fate of childless couples, the main concern in the present study is to design and development of a rulebased inferencing system for infertility diagnosis in women providing the causes of infertility and control measures of the particular problem of infertility which is identified. The proposed study unites the experimental knowledge with the instinctive reasoning skills of a multitude of specialists to aid the infertile women in making the best decision to overcome their problems. The system background starts with the collection of different causes of infertility from infertility specialists and literature related to infertility in women in the Manipur state. Then the acquired knowledge is represented to develop the rule-based inferencing system using JESS. We have implemented here the user interface component using Java, the knowledge base of the system using the Java Expert System Shell (JESS) and the Java IDE of Netbeans 7.0 while the database component is implemented using SQL. The proposed framework can be used as guidelines for infertility diagnosis for women to assists the physicians with their daily practices and women who had infertility problems.

The present study is organised into five headings. The first heading relates to the introduction and objectives of the research problem. It gives a clear picture of the alarming size of the population affected by infertility issues in women in the context of global, national and particularly in Manipur. It also relates to the main concern of the study which is to design and development of a rule-based inferencing system for infertility diagnosis in women using JESS providing the causes of infertility and control measures of the particular problem of infertility which is identified. The second heading is concerned with the application of the expert system in the field of medical diagnosis with some examples of medical diagnosis expert system. The third heading is organised into the development of knowledge base development using JESS with the steps of problem definition, knowledge acquisition, and knowledge representation. The fourth heading focuses on the architectural framework and different components of the rule-based inferencing system for infertility treatment in women. It describes the different components namely User Interface, Explanation Facility, Knowledge Base, Working Memory and Database component of the system. The last and fifth heading summarizes the research contribution by highlighting the conclusion of the research work, and also includes the discussions on future research directions.

\section{RULE-BASED InFERENCING SySTEM In MEDICAL DiAgNOSIS}

Rule-based inferencing system is one of the most common applications of Artificial Intelligence. It is a computer program normally composed of a knowledge base, working memory, inference engine and user interface. The application areas of such a system are rapidly increasing and medical diagnosis is also one of the major application areas. The proposed system is for the infertile women to early identify and diagnose the related symptoms that may contribute to infertility which became an important factor of childlessness because infertility has severe consequences for women's wellbeing particularly. In Indian culture, women typically gain prestige and security in their husband's homes only after they have given a birth child. Many domain-specific expert systems are being used in medical diagnosis in different levels. Some of the examples of expert system applications in medical diagnosis are described below. 
MYCIN was the first and prototypical example of a large family of expert systems that were developed in the 80 's of the $20^{\text {th }}$ Century. MYCIN is a system that gives advice about treatment of infectious diseases. It uses rules to reason in a backward manner: starting with a goal to derive the identity of the organism causing the disease, it constructs a chain of rules that ultimately prove that the hypothesis using observations made by the user. "Relational Model Based MultiDisease Diagnosis Expert System," is a biomedical expert system to help the users in the diagnosis of common human disease and the diagnosis is based on patient's symptoms [23]. The system is implemented using Oracle \& Java technology, and presently encompasses 31 different symptom categories and total 166 symptoms under them. Another expert system "Rule-Based Expert System for Diagnosis of Neuromuscular Disorders," is a system implemented as a rulebased expert system using JESS for the diagnosis of Cerebral Palsy, Multiple Sclerosis, Muscular Dystrophy and Parkinson's disease [24]. It can aid and support the patients suffering from neuromuscular diseases to get an idea of their disease and possible treatment for the disease. "An Expert System Framework for Infertility in Women using Web Based Application," is an application of the expert system in the domain of infertility diagnosis of women [25]. The proposed framework intended to enhance the existing tools used to identify and diagnose infertility problems. Their system can be used as a guideline to develop a web-based expert system for infertility diagnosis. There is another system "Expert system for diagnosis of women's menstrual cycle using natural family planning method," which contributes the algorithm and userfriendly software for fertility diagnosis [26]. This paper presented the algorithms for the expert system supporting the women fertility diagnosis and evaluation of pregnancy achievement. Another system has proposed that "Infertility Expert System Architecture for Early Diagnosis," which is taken up to overcome the problem of infertility in women [27]. The main objective of the study is to develop an architectural framework of infertility expert system for early diagnosis of infertility in men and women. This will help a couple to get an early information and guidance regarding their infertility problem. "An Adoptive Medical Diagnosis System Using Expert System with Applications," is also proposed which is very helpful for the patients that infected with common diseases [28]. This system will give a prescription as a medical expert and also this system is very helpful for rural areas where we have the young medical expert or don't have the medical expert. "A Multilevel Expert System in Medicine," is a system for the diagnosis, prognosis, and monitoring in medicine [29]. These problems are solved with procedural programming and logical programming CLIPS language. This double approach allowed a comparison between different methods of implementing an expert system. A review paper on "Medical Expert System - A Comprehensive Review" represents a comprehensive study of medical expert systems used for diagnosis of various diseases [30].

\section{KNOWLEDGe BASE DeVElopMENT OF THE System}

The idea behind the development of such a rule-based inferencing for infertility diagnosis in women is that it can be used as the medical diagnosis for infertility in women and enable to benefit from the knowledge of infertility experts. Knowledge base development is the most important part in the development of this system because the system depends on its knowledge base. Knowledge base development in this proposed rule-based inferencing system is taken care with Java Expert System Shell (JESS) and the Java IDE of Netbeans 7.0. Knowledge base development here in the proposed system is divided into two major modules namely the diagnosis module and remedies module. These two modules have different tasks. For diagnosis module, it will perform the diagnosis task by prompt questions and then provide diagnose result to the user. 
Here the diagnosis module has again two sub-modules, which are sign and symptoms sub-module and results sub-module.

The sign and symptoms sub-module require the user to answer several questions for the system to present a diagnosed result. The orders of the questions in the system are based on its importance and priority. Simple questions are formed to ease the user in answering the questions and not to be hesitated or confused. Meanwhile, the result sub-module is presented when the system found a conclusion based on the answers given. The remedies module presented brief prescription and recommendation of possible solutions and treatments towards the infertility problem in women. In the process of knowledge base development using JESS, the following steps are recommended and the comprehensive description of each of them is presented in the following.

\subsection{Problem Definition}

The first thing we have to do in the development of knowledge base is to define the problem and understand the major characteristics of the problem that we have to solve by inferencing in the system. The input problem here in the rule-based inferencing of infertility diagnosis in women is regarding the problems which are the reasons of infertile during their married life. Many different health issues can affect woman's ability to get pregnant. Some of the more common problems we are discussing are: Hormonal problems, Endometriosis, Polycystic Ovary Syndrome (PCOS), Tubal Infertility, Damaged Ovaries, Ovulation disorder, Pelvic Inflammatory Disease (PID), Infection, Behavioural Factors, Environmental and Occupational Factors, Other variables that may cause infertility in women etc. The input problem is structured to represent in the form of rules in knowledge base for providing diagnose result and remedies if there is.

\subsection{KNOWLEDGE ACQUISITION}

The rule-based inferencing process involves the elicitation of the expertise knowledge of human expert(s) and the translation of the knowledge thus attained into a machine-executable form. Knowledge acquisition is classically referred to as the bottleneck in the development of inferencing system because the resulting system depends on the quality of the underlying representation of expert knowledge. This is the process to extract the structured and organised knowledge from various sources of human experts and is also consider as the process of adding new knowledge and to change which was anonymously acquired to the knowledge base. It is mainly used in the system development. The purpose of knowledge acquisition is to elaborate the capability of the system to improve the specific task of the performance consisting of facts, rules, concepts, procedures, heuristics, formulas, relationships or other useful information. Knowledge is acquired for this study from the experts in the field of infertility diagnosis which includes Reproductive Endocrinologist or Urologist, or in some cases Obstetrician/Gynaecologist that have expertise and knowledge about infertility problems and its treatments. The acquired knowledge is analyzed and then processed to obtain the best conclusion for the problem. Once the knowledge about infertility in women is acquired from different domain experts and resources, the acquired knowledge is then processed for converting in the form of rules for representing the knowledge in the knowledge base. Thus knowledge acquisition process in the proposed system is carried out until the best conclusion is obtained. 


\subsection{KNOWLEDGE REPRESENTATION}

Knowledge representation is to capture knowledge by the knowledge engineer and is the faithful representation of what the expert knows. The success of a rule-based inferencing system depends on choosing the knowledge representation scheme. There are several techniques of knowledge representation such as First-order logic, semantic networks, Frame-based and Production rules etc. Different knowledge representation formatting have emerged and drive towards efficient knowledge representation has also led to the development of knowledge representation languages like PROLOG, CLIPS, JESS etc. Knowledge acquired from domain experts and related literature of infertility diagnosis is represented in the form of rules using Java Expert System Shell (JESS) and it is the last phase of knowledge base development. JESS is a rule base language for specifying expert systems which can be tightly coupled to code written in the powerful, portable Java language. JESS is architecturally a production system executing a rule-based program and is a scripting language written entirely in Sun Microsystems's Java language. JESS is, therefore, a declarative language.

In this system, the sign and symptoms of a particular problem of the infertility are presented with a list of questions which the user has to answer yes or no. With the help of the questions, the sign, and symptoms of the particular problem of infertility are acquired. The knowledge base is then searched for matching the sign and symptoms of the infertility problem stored in the working memory with those already represented in the knowledge base by using the backward chaining of the Rete Algorithm. Once the system finds the appropriate match, the diagnose result is shown to the user along with the recommendation of possible solutions and treatments towards the infertility problem.

An example of how the knowledge is represented in the knowledge base about Ovulation Disorder problem of infertility using JESS is given below.

(defrule Ovulation-Disorder

(declare (auto-focus TRUE))

(answer (ovulation infrequent) (text yes))

(answer (ovulation absent) (text yes))

(answer (period oligomenorrhea) (text yes))

(answer (period amenorrhea) (text yes))

(answer (hormone imbalance) (text yes))

$\Rightarrow$

(printout t "The infertility problem in the woman is Ovulation Disorder" crlf)

Again we have represented Polycystic Ovary Syndrome (PCOS) in the knowledge base as shown in the following.

(defrule Polycystic-Ovary-Syndrome

(declare (auto-focus TRUE))

(answer (is obesity) (text yes))

(answer (elevate insulin-level) (text yes))

(answer (is resistance-insulin) (text yes))

(answer (is oily-skin) (text yes))

(answer (is dandruff) (text yes)) 
(answer (is skin-discoloration) (text yes))

(answer (high cholesterol-level) (text yes))

(answer (growth excess-hair-on-the-body) (text yes))

$\Rightarrow$

(printout $\mathrm{t}$ "The infertility problem in the woman is Polycystic-Ovary-Syndrome(PCOS)" crlf)

Thus we have represented the acquired knowledge of infertility in women in the knowledge base using Java Expert System Shell (JESS) in the above form.

\section{ArChitectural Framework Of RULE-BASED INFERENCING SySTEM}

To design the rule-based inferencing system for infertility diagnosis in women, initially, we have to identify and understand the problem properly. To understand the functions of the system, it is very necessary to look the architecture of the system and to examine the different components that contribute to present the expert's knowledge in such a system as shown in the figure 1, which mainly highlights the important components of the system such as: User Interface, Knowledge Base, Working Memory, Inference Engine and Databases. The architecture will be slightly different for every problem domains. This architecture will describe the most common components required for the system for infertility diagnosis in women. The components are discussed separately below:

\subsection{USER INTERFACE}

It is one of the basic components of our system which is responsible for the communication mechanism where the user can easily query and get recommendation/response from it. The design of user-friendly interface in our system is very necessary so that people can communicate with the system in natural ways. In addition to being highly interactive, it also provides a transparency of dialogue, whereby some form of an explanation facility indicates the inference process that is being used. The User Interface component in this rule-based inferencing system for infertility diagnosis in women is implemented using Java.

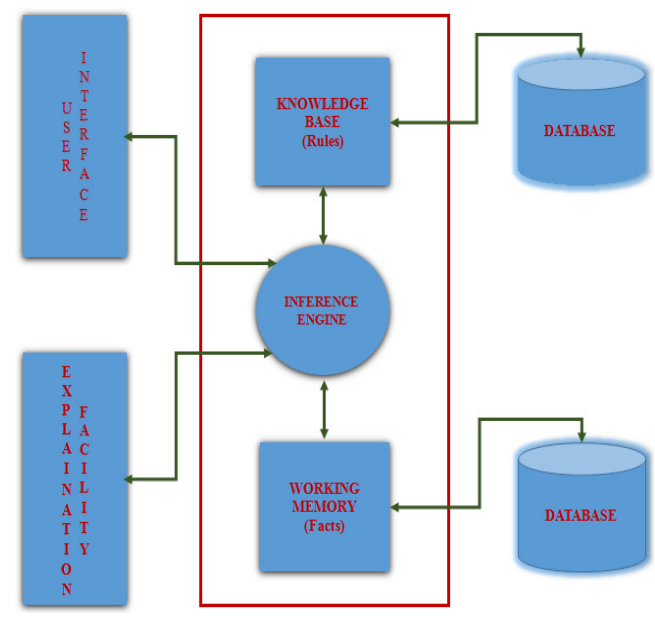

Figure 1: Expert System Architecture 


\subsection{EXPLANATION FACILITY}

It explains the user about the reasoning process of the system. By keeping the track of the rules that are fired, the explanation facility of this system gives a chain of reasoning for a particular problem of infertility in women that led to a certain conclusion. So we called the explanation facility in this system sometimes a justifier. This feature makes a huge difference from other conventional systems. We have implemented here the explanation facility with the help of JESS trace based explanation which is explaining the inferencing on a specific data set. With the help of this, the user or decision maker can understand how the system arrives at certain conclusion or result through the user interface provided by the inference engine. What we get the recommendation or related answer must be related to facts and rules of infertility in women.

\subsection{KNOWLEDGE BASE}

Knowledge base component holds the problem-solving knowledge of expert(s). It consists of specific knowledge and general knowledge. Specific knowledge is those correspond to the data that has been acquired and it is usually stored in a structured way. The knowledge could be presented in the form of rules and sub-rules. We can think of general knowledge as the intentional part of the knowledge base. Knowledge base, here, is the collection of rules which is converted from specific knowledge related to the causes of infertility in women. All the knowledge elicited from domain expert(s) is transformed in the form of rules in proper and efficient manner. Since the knowledge is continually changing and expanding it is considered to be very important that the knowledge base is clearly structured and can easily be modified if required to do so. Building an effective knowledge base requires timely planning, accounting, and organisation of knowledge structure. The key to the knowledge base is how the elicited knowledge is represented. The knowledge acquired from the expert(s) has to be represented formally. Thus knowledge representation deals with the structuring of the information, manipulation of the information and knowledge acquisition. The knowledge base component of the proposed system is structured in this way by using the Java Expert System Shell (JESS) along with the Java IDE of NetBeans 7.0.

\subsection{WORKING MEMORY}

Working memory is used to store the collection of all the facts related to the problem domain of infertility in women which will later be used by the rules in the knowledge base. It contains all the pieces of information of infertility in women in which the system is working with. It can hold both the premises and conclusions of the rules in the knowledge base. It is used by the inference engine to get facts and match them against the rules in the knowledge base. Typically, the rule engine maintains one or more indexes, similar to those used in relational databases, to make searching the working memory a fast operation. The facts may be added to the working memory again by applying some rules.

\subsection{INFERENCE ENGINE}

Whatever the result provided by the expert system must be decided by the inference engine. This part of the system can be considered as the Brain of the system like CPU acts in a computer. It makes the system able to conclude or decide the most appropriate result which we used the term inference in this context. To implement the inference engine in this system for infertility diagnosis in women, we have used the Java Expert System Shell (JESS) and Java IDE of 
Netbeans 7.0. The rules in the knowledge base which is represented in the JESS file is called in the Netbeans environment along with the facts in the working memory. Then by using the Rete Algorithm, the inference engine is matching the sign and symptoms facts in the working memory with those represented rules of infertility diagnosis in the knowledge base. When the JESS rules are executed in Java, JESS library files are also to be loaded into the class path of Java. In JESS, there are two JAR files present namely JESS.jar and jsr94.jar. These two jar files should be included in order to execute a Java file which has JESS commands embedded in it. Instances of JESS rule engine will be created in Java code and can then be reused. JESS rule engine is having special API to execute rules.

For creating an instance of JESS rule engine,

engine $=$ new $\operatorname{Rete}()$;

engine.reset();

engine.batch("infertility.clp");

Here, "engine = new Rete();" is an instance of Rete engine by which JESS is embedded with Java and engine.batch("infertility.clp"); is to load the diagnosis rules of infertility of women.

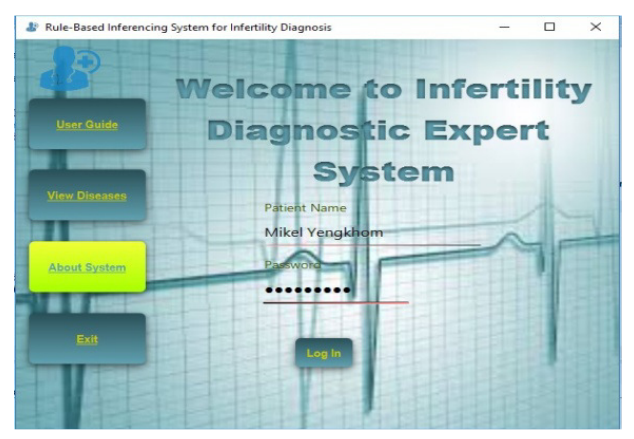

Figure 2: Login Interface

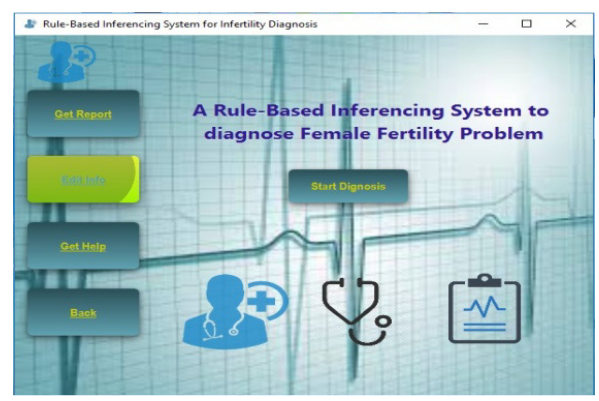

Figure 3: Main User Interface

After implementing the JESS in Java, the sign and symptoms facts in the form of data are loaded into the working memory which is shown in the following.

Database $=$ aDatabase;

engine.addAll(database.getSymptoms()); 
International Journal of Artificial Intelligence and Applications (IJAIA), Vol. 8, No. 1, January 2017

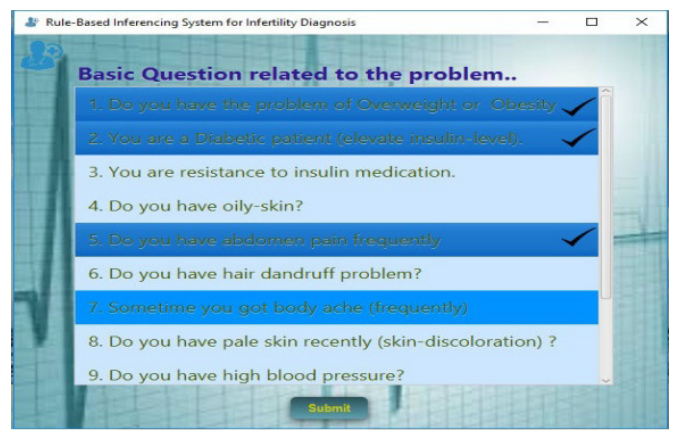

Figure 4: Diagnosis Dialogue Interface

After completing the above process, the run command is given and then all the rules matching the facts will be fired.
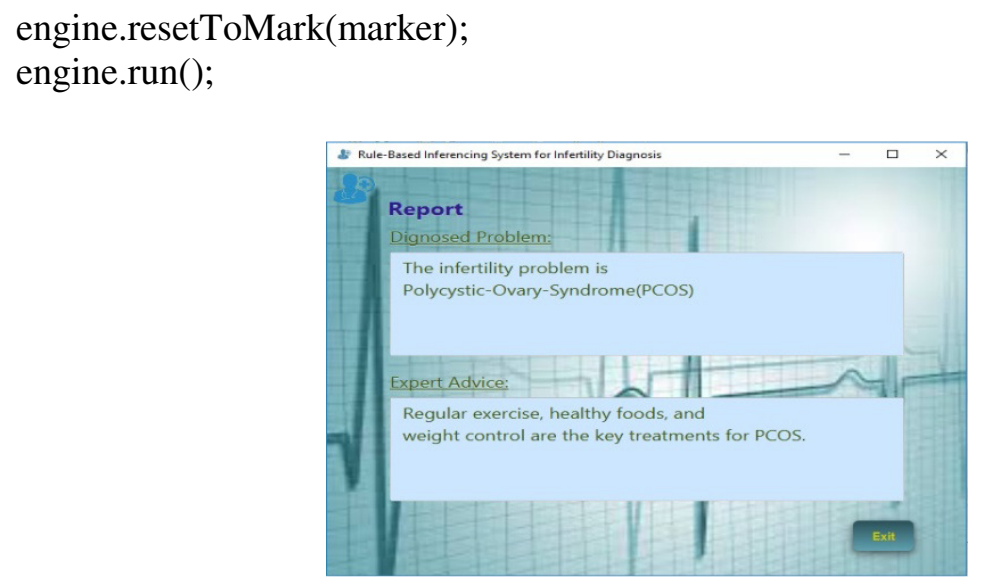

Figure 5: Diagnosed Problem of Infertility

Here, engine.resetToMarker(marker); is to remove all the previous facts/data and engine.run(); is to fire the rules that apply to the given facts/data.

In this way, the inference engine of the system is working and process user's choice and makes a suitable decision.

\subsection{DATABASE}

The database component of the system is used to integrate the knowledge base and working memory components of the system with the database management system. There are two approaches to integrating expert systems and databases - weak and strong coupling. We have used the strong coupling of integrating the proposed system and database which allows one to multiple reads and modify the content of the database. We have stored the rules in the knowledge base and facts in the working memory of the system in the database using SQL. The main reason for integrating the knowledge base and working memory with the database management system is constantly increasing the size of the information to be managed by the system. 


\section{CONCLUSION}

This paper presents the architecture, design, and development of a rule-based inferencing of infertility diagnosis in women which is used to diagnose the common infertility problems in women in Manipur. The system is a rule-based system and the rules are contained in the knowledge base of the system implemented using Java Expert System Shell (JESS). It offers the solution to how a particular problem of infertility in women can be handled by a reasoning approach based on its knowledge base and is easy to be accessed by the users of the system. Manipur is facing the shortage of medical experts in the field of infertility treatment. Due to the shortage of medical expert in the field of infertility, there are getting a huge queue of patients in hospitals and private clinics. So, the "Rule-based Inferencing of Infertility Diagnosis in Women using JESS" can be a substitute of the above problem. This is very useful to diagnose infertility problem of the patient and prescribe the good prescription to them as a human infertility expert. From the above study, it is concluded that this "Rule-based Inferencing of Infertility Diagnosis in Women using JESS" can be applied anytime, anyplace, any hospital to provide the medical prescription for infertility treatment. The paper shows the essence of such a rule-based inferencing system for solving infertility problem in women. This enables the user to diagnose their own infertility problem without referring to infertility experts. There are future scopes for this rule-based inferencing for infertility diagnosis. It will be implemented for the diagnosis of infertility in men. It will also be implemented for the causes of infertility among women of different communities in Manipur because the menarche, menopause and the reproductive span among women of the different communities in Manipur are different.

\section{REFERENCES}

[1] Neha Bhuyan; "Infertility A Growing Problem," Hindustan Times, Mumbai, February 08, 2010, 00:54 IST.

[2] Pratibha Chauhan; "Childless? It's stress!" Tribune News Service, Monday, January 19, 2004, Chandigarh, India.

[3] Dr. Indira Ganeshan; "Why Infertility is on a rise in India It is no longer an urban phenomenon and not just in Women," dailyO, LIFE, 05-02-2016.

[4] RANJANA DIGGIKAR; "IN FIVE YEARS, ALMOST 30\% RISE IN INFERTILITY CASES, SAY EXPERTS,” TIMES NEWS NETWORK I NOV 24, 2012, 12.01 AM IST.

[5] Sujata Ganguly, Sayeed Unisa; "Trends of Infertility and Childlessness in India: Findings from NFHS Data," F, V \& V in ObGyn, 2010, 2(2): 131-138.

[6] Usha Ram; "Levels, Differentials and Spatial Patterns of Childlessness in India," IIPS Working Paper No. 1, April 2010.

[7] Sumita Dutta, Ranjit Guha; "A Clinico-Anatomical Study On The Common Etiological Factors Pertaining To Primary Infertility In Females Using Some Common Investigative Procedures," J. Anat. Soc. India 56 (2) 14-17 (2007)

[8] ROSHNI MAHESH, "INFERTILITY RATE AMONG INDIAN COUPLES ON THE RISE, SAYS SURVEY,” INTERNATIONAL BUSINESS TIMES, SEPTEMBER 20, 2013, 14:55 IST.

[9] T S Syamala; "Infertility in India: Levels, Trends, Determinants and Consequences," (c) 2012, Copyright Reserved. The Institute for Social and Economic Change, Bangalore; ISBN 978-81-7791140-4

[10] Aiswarya P.V. and G. K Moli; "Childlessness among Women in India," Health and Population Perspective and Issues 35(3), 114-131, 2012.

[11] S. Jibonkumar Singh; "Ethnic Variation in Fertility Patterns among Four Communities of Manipur." Journal of Human Ecology, International Interdisciplinary Journal of Man-Environment Relationship; J. Hum. Ecol., 20(1): 1-9(2006) 
[12] Mohammad Asghar, Benrithung Murry, and Kallur Nava Saraswathy; "Fertility Behaviour and Effect of Son Preference among the Muslims of Manipur, India." Hindawi Publishing Corporation, Journal of Anthropology, Volume 2014, Article ID 108236, 5 pages

[13] G. Kameih and G. K. Kshatriya; "Fertility and its determinants among the Lamkang Tribe of Chandel District, Manipur." Human Biology Review (ISSN 2277 4424) 5 (1) 2016

[14] L. Khiloni; "Fertility Performance of the Anal Women of Lambung Village, Chandel District, Manipur." Anthropologist, 11(4): 277-280 (2009)

[15] Ginzamang T. Zomi; "Effect of Son Preference on Fertility Regulation in Manipur." Quest Journals, Journal of Research in Humanities and Social Science, Volume 2 Issue 11 (2014) pp: 46-50, ISSN(Online) : 2321-9467.

[16] Dr. N. Sharat Singh, Th. Manglem Singh; "Indicators of Differential Tribal Fertility in Manipur." IOSR Journal Of Humanities And Social Science (JHSS) ISSN: 2279-0837, ISBN: 2279-0845. Volume 5, Issue 6 (Nov. - Dec. 2012), PP 22-27

[17] Dilip C. Nath and H. Brojeshwor Singh; "Impact of Son Preference on Fertility Indices in Manipur." International Journal of Management, Economics and Social Sciences 2012, Vol. 1(2), pp. 26 -32. ISSN $2304-1366$

[18] Chingakham Dina, Arambam Karamjit, "Manipuri Women Infertility Suspected on Food," EPao.Net, May 21, 2016.

[19] Uzma Amin, Nilofer Khan, Dr. Imtiyaz Ali Bhat; "Prevalence and Causes of Infertility among women of Jammu and Kashmir," International Journal of Development Research, Vol. 5, Issue, 03, pp. 3771-3774, March, 2015.

[20] Marcia C. Inhorn and Pasquale Patrizio; "Infertility around the globe: new thinking on gender, reproductive technologies and global movements in the 21st century." Human Reproduction Update, Vol.21, No.4 pp. 411-426, 2015

[21] K N Sonaliya; "Infertility: Ongoing Global challenge of new millennium." Indian J Comm Health. 2016; 28, 2: 113-115.

[22] Seyede Batool Hasanpoor-Azghdy, Masoumeh Simbar; "The emotional-psychological consequences of infertility among infertile women seeking treatment: Results of a qualitative study," Iran J Reprod Med Vol. 12. No. 2. pp: 131-138, February 2014.

[23] Chandra Prakash Rathore, Swati Rathore, Jyoti Rathore, Chandra Kala Rathore; "Relational Model Based Multi-Disease Diagnosis Expert System," International Journal of Advanced Engineering and Global Technology, ISSN No: 2309-4893, Vol-04, Issue-03, May 2016.

[24] Rajdeep Borgohain, Sugata Sanyal; "Rule-based Expert System for Diagnosis of Neuromuscular Disorders," Int. J. Advanced Networking and Applications, Volume:04 Issue:01 Pages:1509-1513 ISSN : 0975-0290, 2012.

[25] Norhidaya Sukar, Nor Liyana Mohd Shuib; "An Expert System Framework for Infertility in Women using Web Based Application," International Journal of Information Systems and Engineering (online), Volume 2, Issue 1 (April 2014).

[26] Gizem, Aksahya \& Ayese, Ozcan (2009) Coomunications \& Networks, Network Books, ABC Publishers. Andrzej Urbaniak; "Expert System for Diagnosis of women's menstrual cycle using natural family planning method," Presented on 17th IFIP Conference on System ModeUing and Optimization, July 10-14, Prague.

[27] Nur Syafiqah Binti Ahmad, Nor Liyana Mohd Shuib, El Salwana Mat Surin; "Infertility Expert System Architecture for Early Diagnosis," International Journal of Information Systems and Engineering (online), Volume 2, Issue 1 (April 2014).

[28] Gufran Ahmad Ansari; "An Adoptive Medical Diagnosis System Using Expert System with Applications," Journal of Emerging Trends in Computing and Information Sciences, Vol. 4, No. 3 Mar 2013, ISSN 2079-8407, (C2009-2013 CIS Journal. All rights reserved.

[29] Liviu Octavian Mafteiu-Scai; "A Multilevel Expert System in Medicine," International Journal of Academic \& Scientific Research, Vol. 1, Issue 2 No. 2, 2013 pp. 1-6.

[30] Rimpy Nohria; "Medical Expert System- A Comprehensive Review." International Journal of Computer Applications (0975 - 8887) Volume 130 - No.7, November2015 
International Journal of Artificial Intelligence and Applications (IJAIA), Vol. 8, No. 1, January 2017

\section{AUTHORS}

Khumukcham Robindro is currently working as an Assistant Professor in the Department of Computer Science, Manipur University. He joined department on $5^{\text {th }}$ November, 2014. His research interest includes Intelligent Systems, Knowledge Discovery, and Machine Learning etc.

Kshetrimayum Nilakanta is research scholar in the Department of Computer Science, Manipur University. His research interest includes Intelligent Systems, Knowledge Discovery and Machine Learning.
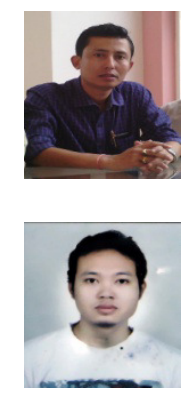